\title{
Caste and Counselling Psychology in India: Dalit Perspectives in Theory and Practice
}

\author{
Meena Sawariya'
}

\begin{abstract}
This paper is written from the perspective of a Dalit counselling psychologist and aims to provide an understanding of the exclusion of Dalit perspective in the theoretical as well as therapeutic domains of psychology as a discipline. It aims to elaborate on the impact of caste on the internalised-self of psychologist as well as their client and how it influences the whole process of learning as well as practice. It identifies the gaps in this field and suggests a revision and reformulation of its course and training programmes so that the closed doors can be opened for all. Further it addresses the various dyads of relationships in therapeutic alliance that can be possibly influenced by caste-based oppression in social life. The paper is highly concerned with the unaffordable and inaccessible nature of clinical settings and the persistent ignorance of the mental health concerns of Dalits. In this paper significant issues like the sense of disconnect, lack of dialogical spaces, and dehumanised processes have been explored in detail. Expressing the hope that there will be a possibility of revisiting and reformulation of theoretical orientations and philosophical frameworks, the paper calls for adequate attention towards the Dalit perspective in counselling psychology to envision egalitarianism in reality.
\end{abstract}

\section{Keywords}

caste, psychology, therapeutic training, mental health, Dalit, counselling

\section{Introduction}

In the world of academic discourse, the persistence of caste as lived experience has been studied significantly from sociological and political perspectives (Guru, 2012; Ilaiah, 2002; Geetha, 2012; Rege, 2003). Psychology offers an important alternative to understand the lived experience of caste in people's lives. However,

\footnotetext{
'Doctoral Scholar, School of Human Studies, Dr. B. R. Ambedkar University, Delhi, India Email: meena.audI7@gmail.com
}

(C) 202I Meena Sawariya. This is an open access article distributed under the terms of the Creative Commons Attribution License, which permits unrestricted use, distribution, and reproduction in any medium, provided the original author(s) and source are credited. 
psychologists in India are yet to seriously engage with caste, and most importantly, yet to reflect on the influence of caste on the theoretical frameworks and practices within the discipline. Majority of psychological research remains oblivious to the deeply ingrained caste biases within the theoretical discourse and practices within the discipline. Davar (1999), in this context, offers an interesting critique of nature and practice of psychoanalysis in India as being dominated by elite upper caste Hindu professionals. In 2002, an interdisciplinary seminar held in Pune on 'Caste and Discourses of the Mind' offered counter discourses of the mainstream ideologies and created space for discussion on 'inner world' and its relationship with caste discourse. It was a significant development in the field of social sciences that considered both theoretical and clinical dimensions enclosed with caste experiences (Davar and Jadhav, 2002). Further, this theoretically disguised world of social sciences, in general, was questioned by Guru and Sarukkai (2012) in their work - The Cracked Mirror. This work offers a critique of the cultural hierarchies' dominant in academic structures, its adverse impact on the production of reflective knowledge and addressed the need for building a category of experience of 'being' Dalit to do the theory. He further addressed the significance of egalitarian principle in reconstructing social sciences observing that it basically interrogates the hierarchical division that suggests that some are born with theoretical spoon in their mouth and a vast majority with empirical pot around their neck...third the egalitarian principle would also interrogate the epistemological imperialism that empowers non-Dalits or tribals to launch intellectual expeditions to conquer new epistemological territories that belongs to the Dalits or Adivasis intellectual universe (Ibid, p. 11).

In light of Guru's argument, the academic discourse of Psychology in India as a discipline as well as the practice has inadequately attended psychological processes and exclusion arising out of caste distinctively. Undoubtedly, Psychology in the West has contributed extensively in studying the psychological aspects of gender, religion, age, race, ethnicity, sexuality, and other forms of inequality. There is little effort in the West to recognise and address the influence of caste as a psychological construct and its predominant existence in the practices associated within the field of psychology.

Some emerging contributions addressing psychological aspects of caste, however, deserve special mention. In the field of cultural psychiatry, the representative work by Jadhav, Mosse and Dostaler (2016) interestingly locates the dynamics of caste-based social suffering from psychological-behavioural perspective. Further, Jadhav (2012) asserts that there is a need to decolonise existing theories of mental health in the Indian context as it lacks space for experiences of local suffering and culturally valid orientation. His extensive work on this subject demonstrates the underlying tenets of guru-shishya relationship amongst mental health professionals in the context of Indian culture and its adverse impact on the well-being of marginalised groups (Jadhav, 2011). His recent work further reflects that the language of caste oppression shall be inverted because the social-psychological distress faced by Dalit students still suffers significant disconnect with the existing diagnostic assessment criteria (Jadhav, 2019). Similarly, an emerging body of social-psychological research engaging with caste provides a promising direction. Jogdand, Khan and Mishra (2016) offers a critique of Cotterill and Colleagues (2014) research that validates persistence of caste through social dominance theory in social psychology (Sidanius and Pratto, 1999). Jogdand et al. (2016) in their paper argue that understanding the nature of social hierarchy and oppression within the caste system and Indian society in general remains inconclusive 
without including a focus on the construction and contestation of social categories and social identities (Tajfel and Turner, 1986). Further, in another work, Jogdand (2017) interrogates caste in the space of classroom setting and advocates for the need to recognise 'casteism' as a major stress factor in the lives of Dalit students. His work calls for more theoretical and policy level engagement on the psychological impact of caste. Building on these contributions and extending the academic discourse and practice of psychology, this paper calls for the need to address Brahminical ideologies permeating psychology in India in its theoretical framework, training, and professional practices. The focus on psychology, in general, and counselling psychology, in particular, is intended to provide a context to address the question of caste as a lived experience and its influence on the inner world at the individual and collective levels.

This article thinks through the various attributes of a counsellor which constitute the practice and articulate the depth of disconnectedness from the perspective of a practitioner. The recognition of caste signifies that it is a psychological reality in social life and is further a signifier of power, position, and collective experience. By avoiding caste recognition, we tend to deny that it has inevitable and conceivable effects on human life and this denial further tends to strengthen the silence about inequalities arising from it.

This dilemma has motivated the author to address the alarming need to evolve a theoretical framework in psychology from the lens of a 'Dalit' with an aim to recollect and re-synthesise the deeper understanding of caste and the human mind. It is based on the author's belief that the struggle against caste injustice and nature of 'caste' as a complex problem requires to be recognised by both academicians as well as practitioners. We need to conceptualise the idea of 'experience' from social identity theory to reconstitute our knowledge systems. This theory and knowledge evolving from 'being' a Dalit will be a unique category of our lived experiences. Even the feminist therapeutic process from a multicultural perspective is exclusive as it still lacks the experiences of Dalit feminism. Thus, the flaws in the theory and therapeutic processes are evident, and hence we need to reformulate these conceptualisations in theory. This reformulation shall be informed on the basis of our experiences and our lenses based on hermeneutics, critical, phenomenological, and grounded perspectives, and methods of inquiry. Given the exclusive nature of the theories and therapeutic processes in psychology, in the following sections, the author attempts to provide a comprehensive account of her journey as a Dalit counselling psychologist drawing upon her experiences in the theory as well as therapeutic processes in practice.

\section{My Journey as a Dalit Counselling Psychologist}

Reflexivity in writing one's own journey is not only about describing the standpoint as a counselling psychologist, it shall also be based on account of narrating how I engaged with real life and came to occupy it through my work. During this journey, there were many precursors. But one of the most significant precursors that contributed towards strengthening my perspectives was my presentation on 'Building resilience among Dalit scholars in higher education: negotiating in new normal world' in the year 2020 during an online national seminar. The presentation was followed by a series of questions on 'why only the well-being of "Dalit" scholars' was the central theme of my presentation. There was a sense of 'unacceptability' towards understanding that persistence of caste in vivid forms is a source of stress in the lives of Dalit scholars. 
Thereby it left little space to accept the contextualisation of caste-based exclusion as a 'problem' and its adverse influence on the human mind. My first experience was at the point of entering the discipline of psychology when my inner self started to rebuild itself as a learner by familiarising myself with the theoretical formulations and conceptual frameworks of the discipline. However, even after being part of it, there were multiple uncertainties and a sense of unrelatedness and unfamiliarity in terms of theories, spaces, methods, and even fellow-learners. Although we had a psychology lab yet its constituents like illustrations on walls, noticeboards, gaze, interactions, and testing assessments lacked a sense of belongingness and affective attachment. During that period, I was also getting reconstructed through various events of violence against Dalits taking place in my native region in Rajasthan. My conscience was able to register the lengthy family discussions voicing concern and fear about the vulnerability of women of our community during such incidents. This fear not only induced a gradual spilt in myself but was also the reason behind the construction of my defences in real life.

The aim of it was to cope with my anxieties and protect my self-esteem from being degraded. Repression, for me, was to exclude myself from the painful memories of debates on reservation in student groups and to work on the thoughts which could make me feel connected to the subject. I experienced constant denial by refusing the existence of any problem in front of my family members. This led to my search for those students who were alike and were experiencing common alienation. It was my association with those students that made me/my mind feel worthy, valued, and affectionate. It inevitably gave me a sense of belongingness due to which my inner self found its home in being with them.

However, in the area of professional growth, the impact of external phenomena remained construed by caste. I can recall the day when I applied for a paid internship (quite a hefty amount) in a clinic inside a hospital setting to learn the therapeutic practice. During internship after post-graduation, I was made to sit outside and was told to observe from 'outside'. The doors of 'experiencing' the therapeutic process were closed which restricted my learning. This was my major encounter with the purity-pollution divide inside the clinic where there was an authority imposed on me to accept my presence as a 'contaminated' subject. The experiential sense of humiliation questioned my worthiness in this subject. And for the first time, I realised how even the therapeutic training, methods, and processes had no recognition for my existence as a 'human' and my dignity, worth, and capability as a learner. This experience resulted in the erosion of emotional expressions which further led to distressing thoughts and reactions. The rejection from the doors of society and the doors of clinics brought about double humiliation and painful wounds that can never be healed. These wounds are irreparable resulting from experiencing exclusion for generations inside as well as outside clinics. I was able to relate my experiences of this isolation with Dr. Ambedkar's (1991, p. 285) experiences about disconnectedness in the education system:

Due to caste there is no common plane on which the privileged and the subject classes can meet. There is no endosmosis, no give and take of life's hopes and experiences. This separation has caused the educated to became slaves and created the psychological complex which follows from a slave mentality. But those affecting the privileged class, though less material and 
less perceptible, are equally real. The isolation and exclusiveness following upon the class structure creates in privileged classes the anti-social spirit of a gang. (Ambedkar, 1991, p. 285)

My experiences of isolation and exclusion thus were sourced from the practitioner's behaviour at the doorsteps of a clinic. It reflected rigidity, low approachable behaviour, lack of empathy, lack of understanding and failure to address my concerns, and stressed on strictly learning from 'outside'. Following constant rejection by the practitioner and refusal to let me observe and learn the processes directly (from inside) just like other interns, I finally had to leave to evolve out of the traumatic experience. However, I found that not only were the boundaries and walls (of the clinics) really high but there was no bridge to reach the learning inside 'clinical' settings.

As I came across my exile inside the discipline and its practice, I was able to seek refuge in the civil society organisations that granted me the opportunity to practise and learn my subject, and achieve expertise. I worked with children with special needs and evolved a new understanding of therapeutic relationship building, and addressing their psychological needs. But at the same time, the denial and rejection remained a source of bruised memories as it shifted my domain of professional expertise towards becoming a 'counselling' psychologist. This calls for an inevitable need to attend the unattended experiences of caste inequalities as crucial to all aspects of psychology as a discipline as well as practice.

\section{Exploring the Possibilities of Therapeutic Alliance}

The derivatives of caste, thus, cannot be isolated from the intrapsychic and interpersonal functioning of a practitioner as a whole. But if these derivatives are inescapable, then how can we assure that the therapeutic process will be effective enough? Thus, it is the responsibility of a psychologist to understand the psychological and social construct of a client's identity and be able to incorporate their experiences in the therapeutic processes and alliance.

It is assumed that effective therapeutic alliance is based on an affective relationship between counsellor and client throughout the counselling process. This alliance determines the quality of goal achievement at each subsequent stage of therapy. There are essential conditions like empathy, unconditional positive regard, transparency, and genuineness that are followed at all the stages. Now let us analyse the therapeutic encounters by looking at the three possible dyads representing caste differences inside the clinic:

\section{The non-Dalit psychologist and the Dalit client}

As a part of existing social structures and institutions, the construction of superiority in terms of caste status can exhibit a strong dominance over the positions of power and authority. This dyad may reflect a strong re-enactment of the dominant identity in non-Dalit psychologist and might provoke distinct behavioural dynamics towards the (un)wanted client. For instance, this was reflective when one of my clients - Sumaaya (pseudonym) recalled her past memory during her post-graduation when she used to meet her teacher: 
Standing outside her room every week used to make me shiver with fear of what was going to happen next. As I was unable to communicate to her about my feelings, she referred me to a psychologist. Meeting and talking to him was once again traumatic, but instead of understanding the depth of caste experience with my professor, he termed my condition as if I was the problem and there was some disorder with me. I stopped approaching him after a few meetings and left the institute.

From the above case study, it can be observed that opening up of caste experiences in itself is distressing and fragile. The task of the psychologist here is to be to develop greater awareness, value-orientation, and integration of anti-oppressive behaviour during the therapeutic process. It will be important for them to recognise, reform, and challenge their internalised attitudes and beliefs about caste supremacy. They must focus on building an egalitarian relationship with the client and situate the issue faced by the client in a context. In this dyad, as reflected above, the client might face lots of valid confusion about 'whether the psychologist will ever be able to understand their problem in the caste context? What if there are judgments in line with caste? Will the therapist understand if I reveal my experiences of discrimination? If so, how?'; and so on.

As the individual understanding of the perceived social realities will definitely be different, the psychologist here needs to be more accommodating in accepting the existence of caste inequality as a problem, and needs to avoid blatant generalisations and stereotypes. This recognition is the only way in this dyad in which a sense of mutual understanding and trust can be established. In the absence of such recognition, the therapeutic relationship will be misdirected, distrustful, and uncomfortable for the client. In this dyad, the psychologist should make efforts in recognising the voice of the client as the only way of knowing his condition, and hence honouring it. The importance of self-introspection on the part of psychologists when relating to others is an alarming need of time and space. As Vahali (2015, p. 251) states:

Guilt can be constructive, transformative emotion... which leads to awakening of conscience about something that is missing but not in others but in ourselves and the way we relate to others.

Thus, there might be occurrence of guilt which can make practitioners encounter the truth that 'Why am I unable to relate' or 'empathise' with the client in the context of caste?' The only method that can resolve this is self-exploration and introspection about one's own beliefs, attitudes, and perceptions so that the guilt can be transformed constructively and the practitioners can recognise that something is 'lacking' in their practice, not in others.

\section{The Dalit psychologist and the non-Dalit client}

This dyad is considered as a rare pairing. Nevertheless, this pairing is the true dyad representing the emergence of egalitarian and emancipatory social realities. The situation of a psychologist belonging from 'Dalit' community as an expert in terms of knowledge may present a sense of dilemma in the client's psyche. For instance, I recall the words of a client who told me that 'you don't talk or look like the ones I consulted earlier.' This realm of categorisation of my body image connotating an 
identity or particular quality generates a set of expectations. It evokes a sense of hypervisibility in the mind of client as they start linking body image or mannerisms to the representational power. There are strong possibilities of distancing as well as withdrawal of therapeutic relationship due to the client's caste-based negative attitude and beliefs. However, the presence of egalitarian values in this alliance can be a ray of hope for social change.

\section{The Dalit psychologist and the Dalit client}

This can be seen as a dyad having space for 'healing' in therapeutic relationships. As there is a sense of belonging, there are much more possibilities of trust, genuineness, psychological comfort and warmth, unconditional positive regard, and a high level of empathy. During conversations with my clients, there was greater use of phrases like 'you already know that these things happen and it affects a lot physically as well as mentally,' or 'At least you will not judge me on those things', and so on. It reflects that both the psychologist and client have faced common social realities and there is a high level of awareness about caste discrimination. Thus, there will be more empathy for each other. Here, the psychologist shall contribute a high level of active listening, attentiveness, sharing of trauma, situatedness, and open possibilities of healing for both of them. As both psychologist and client belong to the same social construct, healthy interaction between the two can facilitate progressive collective experience by opening of doors for emancipation and achieving real-life equality for their community.

The aforementioned dyads offer significant clarity about three diverse relationships that may emerge in therapeutic alliance. Moreover, there might be complex transference and countertransference issues related to cultural identity that can also occur during the therapeutic process. Comas-Diaz and Jacobsen (1991) offers a deeper insight about transference and countertransference that might possibly emerge during ethnocultural therapeutic relationship. In his extensive work on the inter-caste transference and countertransference Jadhav (2003) reveals that:

it is accompanied either by over-compliance, extra-friendliness, a denial of caste, or anger and distrust. Sometimes, through a process of collusion, a dalit can suffer from over-identification with caste Hindus

In the light of above observations, the first dyad can be experiences of disconnect in language or verbatims. However, on the other hand, there are also possibilities of being influenced by therapist, being more friendly and overdependent. Similarly, in the last dyad there might be possibilities that the client might consider therapist as an insider and expert who can rescue the client further. In the last dyad, there are also chances of emergence of subjective countertransference where exclusion related experiences of the therapist might influence the process as well. These issues and concerns need to be explored more from culturally validated perspectives in India. It will enhance our focus on rebuilding knowledge about the collective experiences of marginalised individuals and groups which is otherwise reinforced through myths, attitudes, and behaviours situated in specific cultural context. The core question that arises in therapeutic alliance is - how can an individual transform earnestly from being a 'perpetrator' to a 'healer' in the therapeutic alliance. This is certainly going to have an impact on the psychic level in communities because each group internalises 
unconsciously their respective positions as per social hierarchy and of course escaping from it or rising above it will be challenging.

\section{(Un)Accessibility and (Un)Affordability}

There is a myriad of interrelated forces that impact on the relationship between a psychologist and client as most of the times the services are paid in nature. Most of the time, issues and concerns of the individuals and groups on social margins are unwanted, unattended, and undesired in this realm of psychological services. Informed by the perspective of cultural psychiatry, the significant work by Jain and Jadhav (2009) highlights the gaps between community mental health services and the unaddressed local mental health concerns in rural India. Based upon local fieldwork experiences, their work emphasises the need of a culturally relevant framework to understand human suffering and its inclusion in professional education to address the real mental health concerns of people at margins. Thus, there is a strong need of counsellors and psychologists to equip themselves with an understanding of impact of local language and traditions that permeate caste-based oppression and structural exclusion. It needs to be integrated in therapeutic care to effectively address concerns of our students, workers, and communities, as 'clinics' are not only exclusive and expensive but also far more out of the reach and culturally distant. This sense of disconnect is experiential in nature as it exposes the exclusionary approach of 'clinical setting'. When asked about access to clinical help participants in my research, most of them responded that there was a block that restricted them from viewing caste as a source of their distress. In some cases, a brief intervention was offered without even going in-depth about the cause or few were even suggested cognitive behavioural therapy in the very first meeting. Most of the times caste oppression as the 'source' of the problem is left unexplored and neglected. And this is further rationalised by substituting the client's thoughts and behaviour as the source of the problem itself. Though psychology as a discipline has grown selectively but it still lacks supportive dialogues and a caring environment for the subaltern. How many psychologists are there who can engage in making mental health care accessible and affordable for the Dalit communities? We need to address this question seriously in terms of the reasons behind selective areas of psychological practices and therapies, especially when it comes to caste. Can there be a possibility of a network of Dalit psychologists whose services can be made accessible to the Dalit communities?

\section{Transformation of Therapeutic Process}

This section aims to argue that there is an urgent need to transform our practices by locating and integrating our experiences and positionality in the power structures of theories as well as the therapeutic process. Even the 'curriculum' and 'training' parts lack any presence of significant debates and dialogues about caste-related issues. For example, the curriculum of undergraduate courses in psychology in north Indian universities lacks dialogical space to deliberate upon psychological distress that emerges due to caste. Its content evokes a mainstream perception of 'inclusiveness' in context of caste (as a normalised category) without offering any insight into the issues of inner world enclosed with caste discourses. 
There is no broad framework that can reflect on intricacies and complexities interwoven with life experiences of caste inequalities existing in social structures and institutions. It has for long ignored the psychological well-being and mental health concerns of Dalits and hence, absence of this area and its need, is a silent norm. This needs to be challenged and reformulated by including caste as a phenomenon affecting all the stages of the lifespan of a Dalit individual and families adversely. With these inclusions, the curriculum must focus on enhancing critical thinking about the existence of caste inequalities and how to address its annihilation for a better inner self and outer world. We require a specific type of theoretical orientation in psychology which can teach students and trainees about the influence of caste. It must be based on individual experiences and an integrated framework to take into account the sociopolitical dynamics of caste resulting in psychological distress. In the training process, there should be participation of voices of Dalit scholars and academicians in order to develop a training programme inclusive of the Dalit perspective.

Based on the work of Ridley et al. (1994, p. 327) on the development pyramid of the multicultural counselling training programme, the curriculum, as well as training programmes can be designed with an aim to develop an insight about the Dalit perspective in psychology. This will develop the potential to stimulate critical debates, reflections, and thought processes in the context of caste. The philosophical orientation underlying curriculum development and training process shall be based on Dalit perspective so that it can become a part of subsequent stages. There should be a focus on developing a resource base of the Dalit perspectives and theoretical orientation based on these perspectives shall be further conceptualised in the foundational stage itself. It must be clearly recognised and stated that caste-based oppression adversely affects the personal identity, interpersonal development, cognitive, affective, and behavioural aspects of our life. The inclusion of Dalit perspectives in all aspects of learning objectives in curriculum development and training will offer an effective resolution for emancipation and strong ethical commitment for future practitioners.

The learning objectives can be linked to the demonstration of case studies and research that reflects caste-related issues, concerns and also the struggle of the Dalit communities to evolve against oppression. This can be included and put forth as a relevant area of research in later stages. Unfortunately, the culturally validated psychological tests for caste prejudices and related experiences, such as humiliation, are negligible and do not even constitute one per cent of the overall psychological tests constructed in India. This reality reflects the 'narrowness' and limited scope of the discipline when it comes to addressing caste. It calls for a serious consideration on the part of academicians and practitioners in the field of psychology to devise culturally validated psychological tests and real-life methods which can effectively take into account the persistence of caste oppression in social life (Jadhav, 2009). Similarly, during training programmes, in both therapeutic as well as supervision, this process shall constitute the element of looking and introspecting the personal self. It can also develop possibilities of 'rebuilding' of their 'self' in accordance with new insights from reformulated inclusive theoretical orientation, philosophical framework, and principles to guide relationships in the therapeutic process. It is evident that the social justice-based interactions were found to be closely associated with the supervisory alliance as it may lead to the development of the trainee's competence and skills which can prove essential in social transformation (Ivey, 1995; Ladany et al., 1997). 
As discussed above, there should be adequate attention in theory and practice in order to address the mental health concerns and issues of the client who is psychologically distressed due to caste. In order to evolve positive well-being in them, psychologists need to have sufficient understanding and acceptance of how oppression and power are manifested within clinics and at every stage of the therapeutic process. There might be strong power differences (as already discussed in the non-Dalit psychologist and the Dalit client) and most of the times the psychologist prefers to be unwilling to attend these issues and accept their responsibilities in addressing and transforming the practice accordingly. There is a scope that there can be a constructive sense of guilt and self-introspection among practitioners that can help them in liberating their minds and clinics from internalised beliefs of superiority and power. Thus, they can work extensively in making therapeutic settings inclusive and non-oppressive. In this context, it is significant for them to recognise the constituents, language, and pedagogy of the oppressed in the voices, speeches, narratives, autobiographies and biographies, memoirs, and stories (Freire, 1970, p. 82-120). These are symbols of pain and struggle which must be recognised and honoured across disciplines. Similarly, it is crucial for practitioners to examine and to be aware of the internalisation of oppressive attitudes within the self. And also to deliberate upon how these may be possibly dealt with for an egalitarian therapeutic alliance. In this context, Ivey (1995, p. 59) emphasises that there is a need for practitioners and therapists to work in alliance with the social justice perspective aiming towards liberation. In Indian psychology itself, there is no emphasis on awareness about the oppressive environments constructed by caste inequalities and emancipatory struggles by the Dalit communities. Contending this chosen erasure in the domain of psychology, I would like to put forth the strong argument by Vera and Speight (2003, p. 270) that:

Without an explicit emphasis on ending oppression, counselors may misconceptualise (or underemphasise) major determinants of (and therefore solutions to) problems that compromise the well-being of marginalised communities.

Thus, there must be a strong commitment by the practitioners in the field of psychology to address these issues and concerns in theories as well as therapies. Without such commitment, transformation in this field will never be inclusive and collective. In order to enforce such a commitment, special consideration must be given to include 'no discrimination on the basis of caste' in the code of ethics followed by Indian psychologists. It is essential because when we practice psychological techniques, we motivate our clients to confront their prejudices, fear, and anxieties and facilitate developing a belief in them that they can work upon the possibility of healing. This process equally applies to all academicians and practitioners in the context of caste-based dynamics and its impact on human functioning. We should encourage this inward-looking process in academic engagement and training processes. It will annihilate the false notions of caste-based superiority from the human mind. My paper thus challenges our present understanding of the learning process in theories and therapeutic processes and presents a critique that can become a foundation for lifelong learning for achieving egalitarianism in the real sense. 


\section{Conclusion}

The therapeutic process cannot be separated from real-life experiences and learning methods in the context of caste. Internal processes are very much influenced by external forces and both integrate in order to shape the dynamics of a therapeutic alliance. Undoubtedly, there are multiple contradictions and perplexities highlighted by embedded silence in discussing caste. Evolving from this silence, this paper articulates that the present frameworks are exclusive in their nature and scope and we need to work on making them inclusive and adaptive in their processes. This is only possible by recognising that caste-based oppression exists and needs to be addressed psychologically as well. We need to explicitly see this in the light of Shweder's (1990) perspective on cultural psychology that emphasises the relevance of studying 'interdependence between human psyche and its local socio-cultural world'. Both constitute and reconstitute each other's evolution and hence cannot be studied distinctively from each other. From this perspective, psychology as a discipline needs to be studied from the 'Dalit psyche evolving in cultural context' intensively in both academic and practice settings to address the lived experiences in this context. Jadhav (2015) in an interdisciplinary seminar on 'Minds of Caste' insists that the perpetrators of caste should reflect upon their own minds and practices rather than building their domination over Dalit autobiographies. Thus, there is absence of self-reflexivity in caste Hindus that needs to be addressed (Jadhav, 2014). The author believes that selfreflexivity, as discussed above, is an essential element of competency as a non-Dalit psychologist in two substantial ways - first, it helps the psychologist to practice inwardlooking process, and secondly, it encourages the psychologist to develop an insight or understanding about narratives of caste-based exclusion and struggles. Both need to be attended adequately as these are highly influential in the whole therapeutic process. Further, the unaffordability of clinics and inaccessibility of mental health services for Dalits have created a huge gap that needs to be addressed and bridged. The ethical considerations shall be reformulated keeping in view the caste-based inequalities and its impact on the whole therapeutic process. We need to rethink, reformulate, and contribute in making the Dalit perspective - a significant section of theoretical orientation and philosophical base in psychology and its practice in the Indian context to attain the goals of egalitarianism and social justice in reality.

\section{References}

Ambedkar, B. R. (1991). Gandhism: the doom of the untouchables. In V. Moon (Ed.), Dr. Babasaheb Ambedkar: writings and speeches, vol. 9. Mumbai: Education Department, Government of Maharashtra.

Anand, S. (2003, April 1). Caste on the couch. Himal South Asia, Retrieved on April 21, 2021 from https://www.himalmag.com/caste-on-the-couch/

Comas-Diaz, L., and Jacobsen, F. M. (1991). Ethnocultural transference and counter-transference in the therapeutic dyad. Am J Orthopsychiatry, 61(3), 392-402.

Cotterill, S., Sidanius, J., Bhardwaj, A., and Kumar, V. (2014). Ideological support for the Indian caste system: social dominance orientation, right-wing authoritarianism and karma. Journal of Social and Political Psychology, 2, 98-116. Retrieved from doi:10.5964/jspp.v2i1.171 
Davar, B. (1999). Indian psychoanalysis, patriarchy and Hinduism. Anthropology and Medicine, 6(2), 177-193.

Davar, B., and Jadhav, S. (2002, December, 14-15). Caste and discourses of the mind - an interdisciplinary seminar organised by BAPU Trust, Pune in collaboration with Centre for Behavioural \& Social Sciences in Medicine, University College London.

Freire, P. (1970). Pedagogy of the oppressed. New York: Continuum.

Geetha, V. (2012). Gender. Kolkata: Stree.

Guru, G., and Sarukkai, S. (2012). The cracked mirror: an Indian debate on experience and theory. New Delhi: Oxford University Press.

Ilaiah, K. (2002). Why I am not a Hindu. Kolkata: Samya.

Ivey, A. E. (1995). Psychotherapy as liberation: towards specific skills and strategies in multicultural counselling and therapy. In J. G. Ponterotto, G. M. Casas, L. A. Suzuki and C. M. Alexander (Eds.), Handbook of Multicultural counselling. Thousand oaks, C.A.: Sage Publications.

Jadhav, S. (2009). What is cultural validity and why is it ignored? The case of expressed emotion research in South Asia. In V. Geest, Sjaak and M. Tankink (Eds.), Theory and action. essays for an anthropologist (pp. 92-96). Diemen: Uitgeverij AMB.

Jadhav, S. (2011). ESRC seminar series, $5^{\text {th }}$ seminar global citizenship as a graduate attribute: cultures of teaching and global well being. UCL. Retrieved on April 21, 2021 from https:// www.academia.edu/26027531/ESRC_Seminar_Series_5_th_seminar_GlobalCitizenship_ as_a_Graduate_Attribute_Cultures_of_Teaching_and_Global_Well_Being

Jadhav, S. (2014). Caste, stigma, and mental well-being. Advanced Study Institute, Division of Transcultural Psychiatry, McGill University, Montreal, Canada. Retrieved on April 21, 2021 from https://www.youtube.com/watch?v=sJvJuYLDhdU

Jadhav, S. (2015). Minds of caste: an inter-disciplinary seminar on how caste identities shape the mind. Retrieved on April 21, 2021 from https://www.ucl.ac.uk/intercultural-interaction/ events/minds_of_caste

Jadhav, S. (2019). Developing an anthropological psychiatry strategy for culturally framed social defeat affecting Dalits in higher education in India. UCL. Retrieved on April 21, 2021 from https://www.researchgate.net/publication/350957990_Developing_an_anthropological_ psychiatry_strategy_for_culturally_framed_social_defeat_affecting_Dalits_in_higher_ education_in_India?showFulltext $=1 \&$ linkId $=607 \mathrm{c} 54 \mathrm{e} 68 \mathrm{ea} 909241 \mathrm{e} 0 \mathrm{c} 631 \mathrm{c}$

Jadhav, S., Mosse, D., and Dostaler, N. (2016). Minds of caste: discrimination and its affects. Anthropology Today, 32, 1-2.

Jain, S., and Jadhav, S. (2009). Pills that swallow policy: Clinical ethnography of a community mental health programme in India. Transcultural Psychiatry, 46(1), 60-85.

Jodhka, S. (2012). Caste, culture, and the clinic. Seminar India, 633, 25.

Jogdand, Y., Khan, S., and Mishra, A. K. (2016). Understanding the persistence of caste: a commentary on Cotterill, Sidanius, Bhardwaj and Kumar (2014). Journal of Social and Political Psychology, 4, 554-570. Retrieved from https://doi.org/10.5964/jspp v4i2.603

Jogdand, Y. (2017). The drowned and the saved: caste and humiliation in the Indian classroom. UNESCO Women Philosophers'Journal, 4-5(3), 304-311. Retrieved on April 21, 2021 from https://unesdoc.unesco.org/ark:/48223/pf0000265538

Ladany, N., Brittan-Powell, C. S., and Pannu, R. K. (1997). The influence of supervisory racial identity interaction and racial matching on the supervisory working alliance and supervisee multicultural competence. Counselor Education and Supervision, 36, 284-304.

Lu, F. G., Lim, R., and Mezzich, J. E. (1995). Issues in the assessment and diagnosis of culturally diverse individuals. In J. Oldham and M. Riba (Eds.), Review of Psychiatry (Vol. 14, pp. 477-510). Washington, DC: American Psychiatric Press. 
Rege, S. (2003). Introduction. In S. Rege (Ed.) In writing caste/writing gender: reading Dalit women's testimonies (pp. 1-9). New Delhi: Zubaan.

Ridley, C. R., Mendoza, D. W., and Kanitz, B. (1994). Multicultural training: reexamination, operationalization and integration. The Counselling Psychologist, 22(2), 227-89.

Shweder, R. (1990). Cultural psychology - what is it? In J. Stigler, R. Schweder and G. Herdt (Eds.), Cultural psychology: essay on comparative human development (pp. 1-44). Cambridge: Cambridge University Press.

Sidanius, J., and Pratto, F. (1999). Social dominance: an intergroup theory of social hierarchy and oppression. New York: Cambridge University Press.

Tajfel, H., and Turner, J. C. (1986). The social identity theory of intergroup behavior. In S. Worchel and W. G. Austin (Eds.), Psychology of intergroup relation (pp. 7-24). Chicago: Hall Publishers.

Vahali, O. B. (2015). 'Could I become human by engaging with your un(der)stated life?': struggles from a psychoanalytical perspective on listening to the mentally ill poor person and the homeless poor. Psychology and Developing Societies, 27(2), 231-253.

Vera, E. M., and Speight, S. L. (2003). Multicultural competence, social justice, and counselling psychology: expanding our roles. The Counselling Psychologist, 31, 253-272. 